\title{
Experiential Training of Hand Hygiene Using Virtual Reality
}

\section{Book Chapter}

Author(s):

Clack, Lauren; Hirt, Christian (1); Kunz, Andreas (1); Sax, Hugo

Publication date:

2021-03

Permanent link:

https://doi.org/10.3929/ethz-b-000475540

Rights / license:

In Copyright - Non-Commercial Use Permitted

Originally published in:

Intelligent Systems Reference Library 196, https://doi.org/10.1007/978-3-030-59608-8_3 


\title{
Chapter 3 \\ Experiential Training of Hand Hygiene Using Virtual Reality
}

\author{
Lauren Clack, Christian Hirt, Andreas Kunz, and Hugo Sax
}

\begin{abstract}
Hand hygiene is widely recognized as an important measure to prevent the transmission of microorganisms that may be involved in healthcare-associated infections. Yet correct hand hygiene remains challenging and healthcare providers struggle to improve adherence. While seemingly straightforward, performing hand hygiene at the right indication using the right technique becomes difficult within the context of complex work processes. We believe that an important barrier to hand hygiene is the invisible nature of microorganisms and delayed expression of healthcare associated infections. This delayed or missing feedback makes it difficult to associate unsafe behaviors with their negative consequences. In this chapter, we describe an application of experiential learning theory to guide the development of a virtual reality hand hygiene trainer in which visual feedback about microorganism transmission and infectious outcomes are introduced in the virtual environment. With this immersive trainer, our aim is to enhance experiential learning and increase intrinsic motivation to perform hand hygiene.
\end{abstract}

Keywords Healthcare - Infection prevention $\cdot$ Hand hygiene $\cdot$ Training • Experiential learning $\cdot$ Virtual reality $\cdot$ Simulation

L. Clack $(\bowtie) \cdot$ H. Sax

Infectious Diseases and Hospital Epidemiology, University Hospital Zurich, Zurich, Switzerland e-mail: lauren.clack@usz.ch

H. Sax

e-mail: hugo.sax@usz.ch

C. Hirt · A. Kunz

Innovation Center Virtual Reality, ETH Zurich, Zurich, Switzerland

e-mail: hirtc@iwf.mavt.ethz.ch

A. Kunz

e-mail: kunz@iwf.mavt.ethz.ch

A. L. Brooks et al. (eds.), Recent Advances in Technologies for Inclusive Well-Being,

Intelligent Systems Reference Library 196,

https://doi.org/10.1007/978-3-030-59608-8_3 


\subsection{Introduction}

Healthcare-associated infections (HAI) acquired during the course of treatment are the most frequent adverse events in healthcare delivery, affecting billions of patients worldwide [35]. In the European Union, the burden of HAI has been estimated at 4.5 million infections, 37,000 attributable fatalities, and 16 million additional days of hospital stay annually [38]. Importantly, multiple systematic reviews and metaanalyses have demonstrated that a significant proportion in the range of $35-55 \%$ of these infections are preventable through the application of evidence-based infection prevention measures $[9,31,33]$. Hand hygiene, for example, is widely recognized as the single most important measure to limit transmission of microorganisms and prevent HAI [1]. Despite its proven efficacy, studies repeatedly report low healthcare provider (HCP) adherence to hand hygiene, often in the range of $40 \%$ [8]. Improving $\mathrm{HCP}$ adherence to hand hygiene is therefore an important priority to avoid preventable infections.

Previous interventions to increase HCP adherence to hand hygiene through education and guideline dissemination have been limited in their success and sustainability [15]. Whereas traditional educational interventions seek to improve adherence by improving knowledge, research increasingly suggests that-rather than limited knowledge-low adherence is more likely due to challenging working conditions that make performing hand hygiene difficult. These conditions include high-workload [22], frequent hand-to-surface contacts [5], lack of visual feedback about contamination [28], missing or poorly located hand hygiene resources [20], and delayed or non-existent feedback about the consequences of missed hand hygiene [28]. To be successful, interventions to improve hand hygiene compliance should be designed to target the actual barriers and support enablers of hand hygiene while considering the context in which hand hygiene occurs [17]. Where such interventions involve training and education, these should be designed based on evidence and theory from education research and social and cognitive sciences to increase their chances of success.

The work presented in this chapter addresses two important challenges to hand hygiene adherence. The first challenge is that microorganisms are invisible. The invisible nature of microorganisms makes it impossible for HCPs to observe transmission dynamics or to know whether transfer of potentially harmful microorganisms to the patient has occurred as a result of missed hand hygiene opportunities. The second challenge is that the negative patient outcomes resulting from missed hand hygiene (i.e. patient colonization or infection) occur only much later in time, and these are impossible to trace back to a single individual yet alone a single behavior. Both, the invisible nature of microorganisms and delayed presentation of patient outcomes, prevent HCPs from associating negative patient outcomes with missed hand hygiene opportunities. These factors prevent the associative learning that would be necessary to increase motivation to perform hand hygiene.

Based on these challenges, we believe that a promising approach to increase hand hygiene adherence is to focus on increasing individual motivation through 
experiential learning [21]. We define motivation as the processes that energize and direct behavior, to include both unconscious and automatic processes as well as conscious choices and intentions [18]. Experiential learning has been defined as the process whereby knowledge is created through the transformation of experience [12]. We propose that by reintroducing vivid visual and emotional feedback, that is, by making microorganisms visible and providing immediate feedback about infectious patient outcomes, we can foster experiential learning and support the non-volitional processes that drive habitual infection prevention behavior.

In the following sections, we begin by reviewing previous works within the field of hand hygiene promotion and discussing the value of virtual reality for healthcare training in general, and hand hygiene specifically. We then present specific design considerations to promote effectiveness of hand hygiene training in a virtual environment (VE), using an adapted experiential learning theory exploratory learning model as a guiding framework [7]. Finally, we demonstrate an application of these design considerations to the development of our experiential hand hygiene trainer.

\subsection{Hand Hygiene-Related Work}

The improvement of HCP adherence to hand hygiene has been established as a global priority and continuous efforts are being made to improve hand hygiene across the world. Traditionally, many of these efforts involve HCP education about when and how hand hygiene should be conducted according to established rules such as the World Health Organization's (WHO) My Five Moments for Hand Hygiene [27]. Such educational interventions have traditionally taken the form of guideline dissemination, bedside education, and hands-on training. With some notable exceptions, these have often failed to achieve significant or sustained improvement in hand hygiene behaviors, likely because such rule-based interventions fail to elicit the motivation necessary to reliably change HCP behavior [17]. Rather than such rule-based approaches, motivation can be improved through associative learning that elicits strong feelings and supports habit formation.

Initial applications using serious gaming [29] and virtual reality [2, 3] for hand hygiene education have also been reported. For example, Sax and Longtin [29] developed a serious game, in which HCPs viewed filmed sequences of a physician caring for a patient, then the gaming HCP decided whether hand hygiene was necessary. Similarly, Bertrand et al. [3] reported a virtual agent-based simulation trainer in which participating HCPs are first taught the rules (i.e. WHO indications for hand hygiene [27]), then participants assess hand hygiene performance of a virtual HCP as a percentage of correctly performed hand hygiene opportunities. While an innovation in the field of infection prevention, these efforts have the same limitation as traditional forms of hand hygiene education in that they use a rule-based approach to increase HCP knowledge about when and where to perform hand hygiene, rather than an experiential approach in which new mental models about best practices are formed on the basis of vivid experience. 
In a parallel stream of research, Lane et al. have employed and patented electronic sensors to automatically measure and prompt hand hygiene behaviors [13]. For example, such sensors can detect whether hand hygiene has been performed upon entering or exiting a patient room and provide alerts to remind hand hygiene [10, 16]. A major benefit of such systems is that they provide real-time behavior-shaping feedback. However, they are currently limited in their ability to detect actual behaviors, and therefore cannot provide feedback on all clinically relevant indications for hand hygiene (e.g. before aseptic tasks).

In the current project, we build on the strengths and overcome the limitations of existing interventions by creating a VE in which HCP behavior can be observed and trained. Specifically, the VE we propose goes beyond previous interventions by providing vivid visual feedback about microorganism transmission and infectious outcomes to encourage experiential learning.

\subsection{Virtual Reality for Experiential Training}

Virtual reality has been increasingly recognized as a promising technology for healthcare training by allowing users to experience and train for situations that would otherwise be difficult to produce in the real world [11,30]. Virtual reality provides a unique opportunity to train both technical and non-technical skills in a realistic, risk-free learning environment. Virtual learning environments can also overcome geographical limitations as training can be conducted outside the confines of a lab or classroom, for example using web-based platforms. Avatars representing colleagues and patients can be introduced to increase realism and practice meaningful interactions. The appearance and behavior of these avatars as well as the overall VE can also be modified in a controlled manner that would be difficult or impossible to achieve in real life settings. A major benefit of virtual reality for healthcare training is that it offers an immersive learning environment where rich experiential learning can occur [7].

For our purposes, virtual reality has the specific advantage of allowing users to experience visual feedback that would be impossible in reality. Given that a VE for experiential hand hygiene training has - to the best of our knowledge-yet to be established, several technical and behavioral research questions emerge. A multidisciplinary approach, bringing together expertise from infectious diseases and hospital epidemiology, psychology, and virtual reality, is indispensable.

\subsubsection{Experiential Learning Theory}

An 'exploratory learning model' that stems from Kolb's experiential learning model has been proposed to guide the development of experiential training using 3D applications $[7,12]$. Both of these models are descriptive rather than analytical, as they 
Fig. 3.1 Stages of the exploratory learning cycle in immersive environments [7]

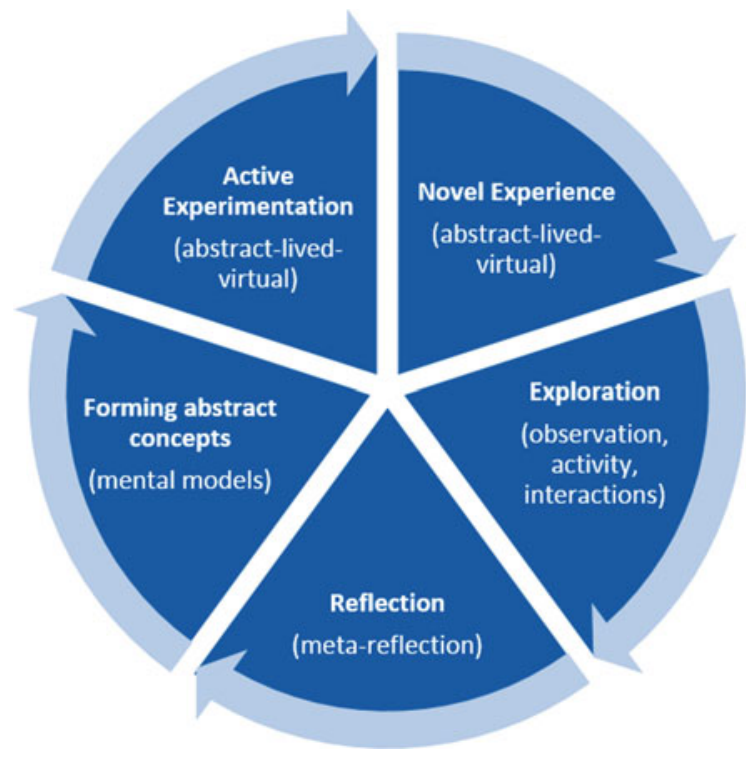

describe an iterative process rather than causal links. Kolb's experiential learning model is composed of four stages that occur cyclically, including: novel experience, reflection, forming abstract concepts and active experimentation in new situations [12]. The exploratory learning model builds on Kolb's experiential learning model in that it takes into consideration the exploratory processes that occur within $3 \mathrm{D}$ and virtual training environments, thereby adding an 'exploration' stage to the model (Fig. 3.1). Central to both of these models is that the learner plays an active rather than passive role in the dynamic learning process and that one should experience all stages of the cycle for effective learning to occur.

To increase the chances that training in the VE will be effective in fostering experiential learning and sustainably changing HCP hand hygiene behaviors, we employ the exploratory learning model [7] as a guiding framework to inform the development of our virtual reality hand hygiene trainer. In the following sections, we describe how the design and functionalities of our trainer are intended to support each stage of the experiential learning cycle. In the following sections, we refer to HCPs who participate in hand hygiene training as 'learners'. The work presented here is based on an advanced experimental prototype that is currently undergoing further refinement $[4,6]$.

\subsubsection{Novel Experience Stage}

The experience stage of the learning cycle begins when a new experience is encountered. The learner is exposed to a novel situation with unfamiliar stimuli, in either 
the real world or a virtual context, of which they must make sense. Given that the VE can be modified in ways that would not be possible in the real world, there exist a multitude of opportunities to introduce new experiences. For our hand hygiene trainer, we have introduced two specific functionalities that are intended to expose HCP learners to novel experiences: microorganism visualization and time-warping.

- Microorganism visualization: Due to the invisible nature of microorganisms in the real world, HCPs are largely unaware of the contamination status of objects in the healthcare environment and they don't receive feedback about how their own manipulations may result in propagation of harmful microorganisms. In our hand hygiene trainer, we make virtual microorganisms visible in the VE so that HCPs can experience the transmission of microorganisms first-hand. Technically, this is achieved by tracking the physical location of the controllers and producing a colored overlay when they come into contact with objects and surfaces in the VE (Fig. 3.2).

- Time-warping: In the real world, healthcare-associated patient infections or colonization with multidrug-resistant microorganisms can only be clinically detected with a significant delay and it is nearly impossible to trace these infectious outcomes back to a specific individual or behavior. For this reason, HCPs are rarely informed about the direct consequences of missed hand hygiene opportunities. In our hand hygiene trainer, we employ time-warping - that is making time in the VE move faster than in reality-to allow HCPs to immediately experience the down-the-line consequences of missed hand hygiene opportunities (Fig. 3.2).

- Behavior and appearance of avatars: Many healthcare tasks are performed by teams or dyads (e.g. fully-trained HCP and trainee) caring for a patient. The social interactions that occur, both among HCPs and between HCPs and patients, play an important role in forming experiences in real and virtual contexts. For example, the presence of a senior physician performing hand hygiene or the belief that hand hygiene is important to your colleagues may increase the likelihood of performing hand hygiene [23]. Further, behavior of the patient, who could remind HCPs to do hand hygiene or react negatively to missed hand hygiene opportunities, is increasingly recognized for the potential to influence HCP hand hygiene behaviors [32]. This is important for our virtual hand hygiene trainer, where we can modify behavior and appearance of avatars representing colleagues and patients to influence the HCPs learning experience.

\subsubsection{Exploration Stage}

Within VEs, the learner is empowered to explore and push the boundaries of what they know while engaging with the environment [7]. The extent to which the training environment allows the learner to explore, as opposed to following a fixed script, is therefore an important design consideration. For our hand hygiene trainer, we have developed multiple care scenarios that serve as storylines to guide the training sessions. The participating HCPs are given a care task to complete that guides their 

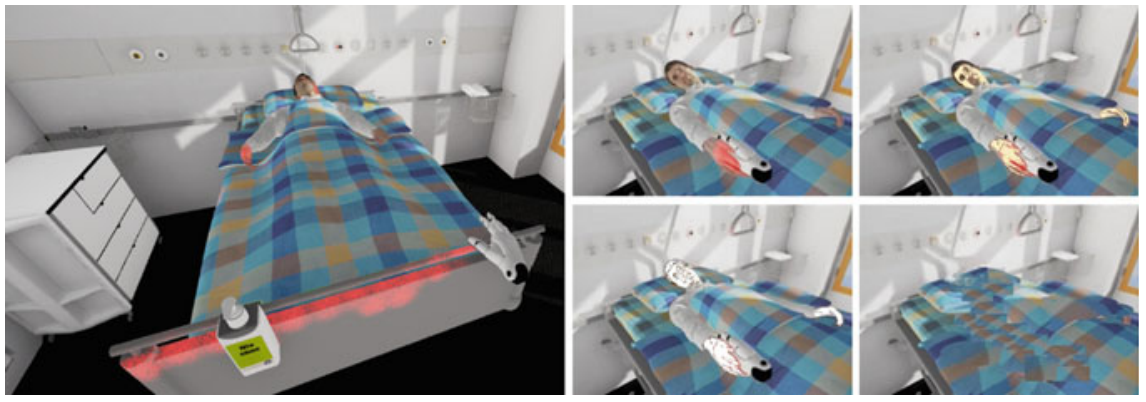

Fig. 3.2 Left: Microorganism visualization on patient bed rail. Right: Visual portrayal of timewarping

activity within the VE. Within the bounds of the care task, HCPs are free to explore and to complete the task as they see fit. To facilitate this exploration, our trainer is designed to allow for real walking within the VE.

- Real walking: Multiple researchers have shown that real walking, as opposed to teleportation using handheld controllers, for navigating VEs improves the user's cognitive map and thus helps understanding the context of the training scenario $[14,25,34]$. Real walking is possible in our current hand hygiene trainer when the physical training space is larger than the VE, otherwise teleporting must be used for navigation (Fig. 3.3). Real walking becomes challenging, however, when the virtual world outsizes the available physical space. Here, a compression of the VE becomes necessary, for example using redirected walking [24] by introducing an unnoticeable mismatch between a user's movement in the real and the VE. Future iterations of our trainer building on our previous work [4, 6, 19, 36, 37] may include redirected walking to improve the immersion for the learners.

\subsubsection{Reflection Stage}

During the reflection stage of the experiential learning cycle, any inconsistencies between the new experience and previous understanding are reflected upon [12]. Reflection is of particular importance for virtual training activities to facilitate the transfer between virtual and lived experiences [7]. Some have argued that the experience itself must be interrupted to initiate a phase of reflection [12]. To foster such reflection, our virtual hand hygiene training is embedded within a process that includes periods of debriefing.

- Debriefing: The debriefing process, whereby individuals reflect on their own practice, is already well established within medical training, for example, following medical simulation [26]. Debriefing after a training scenario offers HCPs an opportunity to reflect on their performance and develop insights that can inform later 
actions [26]. In the current project [4, 6], debriefing takes the form of a live, post-training session in which the HCP learner has an opportunity to reflect on the virtual training experience. During debriefing, the learner may review videoexcerpts from their training exercise and view descriptive data about the extent of contamination that was established as a result of missed hand hygiene opportunities in the VE. The goal of this debriefing is to help learners to understand, analyze, and synthesize how their behaviour influences microorganism transmission and infectious patient outcomes in order to improve future hand hygiene performance.

\subsubsection{Forming Abstract Concepts or Mental Models}

The process of reflecting on new experiences inevitably gives rise to new ideas or leads to modification of one's existing 'mental models' or 'frames' [26, 28]. Mental models can be defined as internal mental images gathered through experience and observation that lead to abstract concepts and representations of how an individual understands the world around her [28]. These abstract concepts or 'models' are then projected onto future experiences and shape future behaviors. Mental models are particularly important in the field of infection prevention, where individual understanding about microorganism transmission and the consequences of preventative measures like hand hygiene will influence the way they behave to prevent infections.

\subsubsection{Active Experimentation in Different Situations}

During the active experimentation stage, also referred to as 'testing in different situations', the newly acquired reflections and mental models are tested as the learner

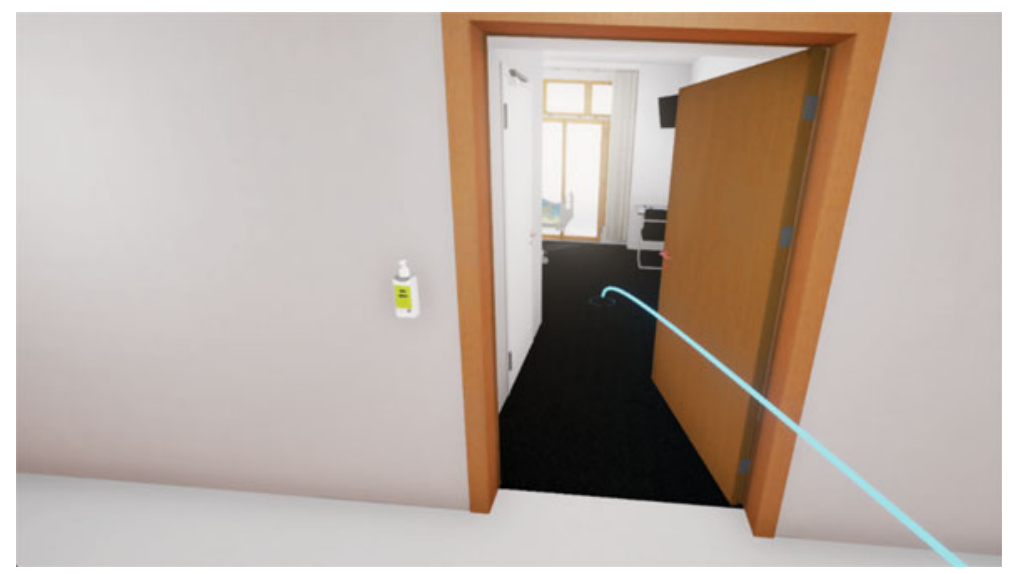

Fig. 3.3 Navigating the virtual environment with teleporting 
is exposed to different situations. For our hand hygiene trainer, this means reentering the virtual training environment after having reflected and formed new mental models about transmission dynamics and the protective role of hand hygiene. Equipped with these new models, participating HCPs reenter the VE with heightened awareness about transmission dynamics. The ultimate assessment of our hand hygiene trainer and subject of future work is to evaluate the extent to which training in the VE translates to improved real life hand hygiene behaviors. We hypothesize that the reflections and mental models developed during virtual trainings and refined during debriefings will translate into an augmented awareness that will remain with HCPs once they return to their daily work, supporting improved hand hygiene performance in the real world.

\subsection{Summary and Future Work}

The exploratory learning model based on Kolb's experiential learning theory was useful for guiding the development of our virtual reality hand hygiene trainer [7, 12]. While using the virtual hand hygiene trainer, HCPs should pass through all stages of the learning cycle: (1) experiencing first hand the visual transmission of microorganism and receiving immediate feedback about infectious patient outcomes together with social feedback from virtual patients and colleagues; (2) exploring the virtual environment through real walking within the bounds of the given task scenario; (3) reflecting on the experience during debriefing; (4) forming new mental models and abstract concepts (e.g. about how HCP behaviors may lead to transmission of microorganisms and the protective role of hand hygiene in preventing infectious patient outcomes); and (5) testing these new mental models through further experiences in new situations. It is worth noting that, while it is important for learners to pass through all stages of the experiential learning cycle, these phases must not necessarily occur sequentially and the learner must not necessarily be consciously aware of the learning process that is occurring. The formation of new mental models, for example, is a largely unconscious process that occurs in everyday life each time a new situation is encountered. We expect that the vivid experience of seeing microorganism transmission and infectious patient outcomes as a result of missed hand hygiene will itself have a strong impact on the learner's internal mental images. By introducing debriefing to the training process, we aim to bring this typically unconscious mental activity to conscious awareness and thereby strengthen the learning process.

The experiential learning approach described in this chapter is consistent with previous research by Nicol et al. [21], who found that vivid vicarious experience, such as personal exposure to an infectious outbreak or caring for a patient affected by healthcare-associated infection, was of greater importance than formal education in explaining HCP hand hygiene behaviors [21]. They highlighted that the emotional impact of having been personally involved in such a situation heightened HCP awareness and resulted in sustained improvements in hand hygiene [21]. With our 
virtual trainer, we aim to elicit the same heightened emotional reaction in a risk-free environment.

An added value of training in virtual reality is that the behaviors and resulting transmission pathways of HCPs participating in training in the VE can be automatically captured and analyzed. This produces a rich data set about HCP behaviors without the need for costly and time-consuming direct observations. The virtual healthcare setting therefore represents a powerful tool for both training and studying $\mathrm{HCP}$ infection prevention behaviors.

This paper describes an innovative approach to improve HCP hand hygiene performance through vivid virtual reality training embedded within an experiential learning process. This trainer aims to improve hand hygiene by reintroducing the otherwise missing feedback about the consequences of unsafe behavior and therefore increase HCP motivation to perform hand hygiene. We believe this hand hygiene trainer has the potential to improve HCP hand hygiene performance in the real world, which will be the focus of future evaluation. Once validated, this system could be adapted for use in other clinical settings (e.g. surgical, ambulatory care) and extended to integrate hand hygiene training into a wide range of clinical processes.

Acknowledgements This project has been generously supported by a donation from Dr. HansPeter Wild to the University Hospital Zurich Foundation. We would further like to acknowledge Dirk Saleschus, project manager, and Marcel Wenger, innovation manager, for their contributions to this work.

\section{References}

1. Allegranzi, B., Pittet, D.: Role of hand hygiene in healthcare-associated infection prevention. J. Hospital Infect. 73(4), 305-315 (2009)

2. Bertrand, J., Babu, S.V., Gupta, M., Segre, A.M., Polgreen, P.: A 3D virtual reality hand hygiene compliance training simulator. In: Scientific Meeting of the Society for Healthcare Epidemiology of America (2011)

3. Bertrand, J., Babu, S.V., Polgreen, P., Segre, A.: Virtual agents based simulation for training healthcare workers in hand hygiene procedures. In: International Conference on Intelligent Virtual Agents, pp. 125-131 (2010)

4. Clack, L., Wenger, M., Sax, H.: Virtual reality enhanced behaviour-Change training for healthcare-associated infection prevention. Front. Publ. Health 45 (2017)

5. Clack, L., Scotoni, M., Wolfensberger, A., Sax, H.: V "First-person view" of pathogen transmission and hand hygiene-Use of a new head-mounted video capture and coding tool. Antimicrob. Resist. Infect. Control 108(6) (2017)

6. Clack, L., Hirt, C., Wenger, M., Saleschus, D., Kunz, A., Sax, H: VIRTUE-A virtual reality trainer for hand hygiene. In: 9th IEEE International Conference on Information, Intelligence, Systems and Applications (2018)

7. De Freitas, S., Neumann, T.: The use of 'exploratory learning' for supporting immersive learning in virtual environments. Comput. Educ. 52(2), 343-352 (2009)

8. Erasmus, V., Daha, T.J., Brug, H., Richardus, J.H.K., Behrendt, M.D., Vos, M.C., van Beeck, E.F.: Systematic review of studies on compliance with hand hygiene guidelines in hospital care. Infect. Control Hospital Epidemiol. 31(3), 283-294 (2010)

9. Harbarth, S., Sax, H., Gastmeier, P.: The preventable proportion of nosocomial infections: an overview of published reports. J. Hospital Infect. 54(4), 258-266 (2003) 
10. Higgins, A., Hannan, M.M.: Improved hand hygiene technique and compliance in healthcare workers using gaming technology. J. Hospital Infect. 84(1), 32-37 (2013)

11. Hoffman, H., Vu, D.: Virtual reality: teaching tool of the twenty-first century? Acad. Med. 72(12), 1076-1081 (1997)

12. Kolb, D.A.: Experiential Learning. Englewood Cliffs (1984)

13. Lane, S., Strauss, K., Coyne, M.: Systems and methods for improving hand hygiene compliance. US Patent 6,975,231 (2005)

14. Larrue, F., Sauzeon, H., Wallet, G., Foloppe, D., Cazalets, J.R., Gross, C., N'Kaoua, B.: Influence of body-centered information on the transfer of spatial learning from a virtual to a real environment. J. Cogn. Psychol. 26(8), 906-918 (2014)

15. Larson, E.L., Quiros, D., Lin, S.X.: Dissemination of the CDC's hand hygiene guideline and impact on infection rates. Am. J. Infect. Control 35(10), 666-675 (2007)

16. Marra, A.R., Zinsly Sampaio Camargo, T., Magnus, T.P., Blaya, R., dos Santos, G.B., Guastelli, L.R., et al: The use of real-time feedback via wireless technology to improve hand hygiene compliance. Am. J. Infect. Control 42(6), 608-611 (2014)

17. Michie, S., Van Stralen, M.M., West, R.: The behaviour change wheel: a new method for characterising and designing behaviour change interventions. Implement. Sci. 6(1), 42 (2011)

18. Mook, D.G.: The Organization of Action. WW Norton (1987)

19. Nescher, T., Huang, Y.Y., Kunz, A.: Planning redirection techniques for optimal free walking experience using model predictive control. In: IEEE Symposium on 3D User Interfaces, pp. 111-118 (2014)

20. Nevo, I., Fitzpatrick, M., Thomas, R.-E., Gluck, P.A., Lenchus, J.D., Arheart, K.L., Birnbach, D.J.: The efficacy of visual cues to improve hand hygiene compliance. Simul. Healthcare 5(6), 325-331 (2010)

21. Nicol, P.W., Donovan, R.J., Wynaden, D., Cadwallader, H.: The power of vivid experience in hand hygiene compliance. J. Hospital Infect. 72(1), 36-42 (2009)

22. O'boyle, C.A., Henly, S.J., Larson, E.: Understanding adherence to hand hygiene recommendations: the theory of planned behavior. Am. J. Infect. Control 29(6), 352-360 (2001)

23. Pittet, D., Simon, A., Hugonnet, S., Pessoa-Silva, C.L., Sauvan, V., Perneger, T.V.: Hand hygiene among physicians: performance, beliefs, and perceptions. Ann. Internal Med. 141(1), $1-8$ (2004)

24. Razzaque, S., Kohn, Z., Whitton, M.C.: Redirected walking. In: Proceedings of EUROGRAPHICS, vol. 9, 105-106 (2001)

25. Ruddle, R.A., Volkova, E., Bülthoff, H.H.: Walking improves your cognitive map in environments that are large-scale and large in extent. ACM Trans. Comput.-Hum. Interact. (TOCHI'11) 18(2), 10:1-10:20 (2011)

26. Rudolph, J.W., Simon, R., Raemer, D.B., Eppich, W.J.: Debriefing as formative assessment: closing performance gaps in medical education. Acad. Emerg. Med. 15, 1010-1016 (2008)

27. Sax, H., Allegranzi, B., Uckay, I., Larson, E., Boyce, J., Pittet, D.: My five moments for hand hygiene: a user-centred design approach to understand, train, monitor and report hand hygiene. J. Hospital Infect. 67(1), 9-21 (2007)

28. Sax, H., Clack, L.: Mental models: a basic concept for human factors design in infection prevention. J. Hospital Infect. 89(4), 335-339 (2015)

29. Sax, H., Longin, Y: Immersive hand hygiene trainer for physicians-a story-based serious game. In: BMC Proceedings, vol. 89, no. O31. BioMed Central (2011)

30. Saxena, N., Kyaw, B.M., Vseteckova, J., Dev, P., Paul, P., Lim, K.T.K., Kononowicz, A., Masiello, I., Tudor Car, L., Nikolaou, C.K., Zary, N., Car, J. : Virtual reality environments for health professional education (protocol). Cochrane Database Syst. Rev. 2 (2016)

31. Schreiber, P., Sax, H., Wolfensberger, A., Clack, L., Kuster, S.: Swissnoso: the preventable proportion of healthcare-associated infections 2005-2016: systematic review and meta-analysis. Infect. Control Hospital Epidemiol. (in press), Presence (2018)

32. Stewardson, A.J., Sax, H., Gayet-Ageron, A., Touveneau, S., Longtin, Y., Zingg, W., Pittet, D.: Enhanced performance feedback and patient participation to improve hand hygiene compliance of health-care workers in the setting of established multimodal promotion: a single-centre, cluster randomised controlled trial. Lancet Infect. Dis. 16(12), 1345-1355 (2016) 
33. Umscheid, C.A., Mitchell, M.D., Doshi, J.A., Agarwal, R., Williams, K., Brennan, P.J.: Estimating the proportion of healthcare-associated infections that are reasonably preventable and the related mortality and costs. Infect. Control Hospital Epidemiol. 32(2), 101-114 (2011)

34. Usoh, M., Arthur, K., Whitton, M.C., Bastos, R., Steed, A., Slater, M., Brooks Jr., F.P.: Walking $>$ walking-in-place $>$ flying, in virtual environments. In: Proceedings of the 26th Annual Conference on Computer Graphics and Interactive Techniques, pp. 359-364 (1999)

35. World Health Organization: Report on the Burden of Endemic Healthcare-Associated Infection Worldwide (2011)

36. Zank, M., Kunz, A.: Where are you going? Using human locomotion models for target estimation. Vis. Comput. Int. J. Comput. Graph. 32(10), 1323-1335 (2016)

37. Zank, M., Kunz, A.: Optimized graph extraction and locomotion prediction for redirected walking. In: IEEE Symposium on 3D User Interfaces, pp. 120-129 (2017)

38. Zarb, P., et al.: The European Centre for Disease Prevention and Control (ECDC) pilot point prevalence survey of healthcare-associated infections and antimicrobial use. Eurosurveillance 17(46), 20316 (2012) 